Check for updates

Cite this: Nanoscale Adv., 2019, 1, 281

\title{
Polysulfide microspheres with chemical modification for generation of interfaces with macroscopic colour variation and biomimetic superhydrophobicity
}

\author{
Zelinlan Wang, ${ }^{\text {ab }}$ Xiaoyu Gao, ${ }^{\text {ab }}$ Gang Wen, ${ }^{\text {ab }}$ Pan Tian, ${ }^{\text {ab }}$ Lieshuang Zhong ${ }^{\text {ab }}$ \\ and Zhiguang Guo*ab
}

Both superwettability and structural colours have attracted considerable attention in recent years. In addition, the combination of structural colours and superwettability could endow materials with broader application prospects. The combination provides a new strategy to design novel functional materials, and there are many studies pertaining to these materials that have been reported in recent years. Herein, a polysulfide (PSF) superhydrophobic coating was synthesized successfully. The PSF superhydrophobic coating possesses excellent superhydrophobicity, oleophobicity for diesel and macroscopic structural colour variation when wetted. The colour is changed when the coating is wetted and it returns to its original colour after drying. In addition, the surface presents better reusability and thermostability which satisfies various daily needs. The PSF superhydrophobic coating can be considered as an excellent candidate for designing wetting responsive materials, and it has enormous application potential in the fields of detection, sensing, anti-counterfeiting and security. For the first time, we present a novel and low-cost strategy to fabricate materials with both superhydrophobicity and structural colour, offering significant insights into the practical application of these functional materials.

Received 6th June 2018

Accepted 23rd July 2018

DOI: $10.1039 / c 8 n a 00011 e$

rsc.li/nanoscale-advances
Another important surface characteristic, structural colour, has also attracted considerable attention in recent years. The phenomenon is attributed to the interference, diffraction and dispersion of light with facile periodic morphologies. ${ }^{15-17}$ Materials with this characteristic have been widely used in many fields such as sensing, ${ }^{18,19}$ bioassay, ${ }^{20,21}$ anti-counterfeiting, ${ }^{22}$ and optical components. ${ }^{23}$ There are many materials with improved optical properties that have been developed and reported to obtain materials with structural colour, including silicon dioxide, polymethyl methacrylate and polystyrene, ${ }^{24-35}$ since the fabrication of structural materials from colloidal spheres is low-cost and facile. And we can adopt a colloidal selfassembly method to obtain a surface with periodic morphology. Therefore, this method has been considered as the mainstream approach to fabricate materials with structural colour. ${ }^{36-39}$ In contrast, the occurrence of structural defects on the surface during the fabrication process limits the application of structural colour. Furthermore, with most polymers, even some traditional optical materials, it is hard to obtain bright and obvious structural colour due to their low refractive index which hinders the generation of a wider (even complete) photonic band gap (PBG). ${ }^{17,40-42}$ And to improve the structural color visibility, black materials are usually added into the photonic ordered arrays to absorb the scattered light on a broadband. ${ }^{43}$ Gianneschi et al. and Kohri et al. adopted this strategy and
${ }^{a}$ Hubei Collaborative Innovation Centre for Advanced Organic Chemical Materials, Ministry of Education Key Laboratory for the Green Preparation and Application of Functional Materials, Hubei University, Wuhan 430062, People's Republic of China. Fax: +86-931-8277088; Tel: +86-931-4968105

${ }^{b}$ State Key Laboratory of Solid Lubrication, Lanzhou Institute of Chemical Physics, Chinese Academy of Sciences, Lanzhou 730000, People's Republic of China. E-mail: zguo@licp.cas.cn 
successfully acquired non-iridescent structural color using black particles. ${ }^{\mathbf{4 4}, \mathbf{4 5}}$ However, the original black color of particles limited the application of the responsive material. Although it can present bright color, it cannot generate an obvious color change for darker colors. The addition of melanin nanoparticles also complicates the preparation technology. But when particles themselves possess a high refractive index, high surface charge, low mass density, and the ability for visible light absorption, this problem is solved perfectly, and the color visibility can be greatly improved.

In order to improve this situation, Zhang et al. made use of persulfide (PSF) polymeric microspheres which possess higher refractive index (as high as 1.858) to construct a surface with structural colour. ${ }^{37}$ The report introduced the production of polysulfide rubber in highly monodisperse spheres with bright colour.

As is known to us, micro-nano-structured morphologies could be created to obtain a superhydrophobic surface. Structural colour can also be obtained through the same morphology. ${ }^{16}$ Additionally, the combination of these characteristics improves the surface properties, such as self-cleaning, selective wetting and colour variation via angle change. The combination provides a new strategy to design novel functional materials, and there are many studies pertaining to these materials that have been reported in recent years. ${ }^{46-52}$ So, it is necessary to explore the possibility of combining superhydrophobicity and structural colour.

Due to the special wettability, materials can only be wetted by specific liquids, which provides the possibility of fabricating materials with selective responsive structural colour, as shown in Fig. 1. And the special colour variation by wetting provides a novel strategy to design anti-counterfeit, responsive and even security materials. Because PSF microspheres can greatly enhance colour visibility, due to their higher refractive index, PSF was chosen in this work to fabricate a material with both superhydrophobicity and structural colour. Up until now, this is the first successful synthesis of a superhydrophobic PSF surface with selective responsive structural colour. In this work,

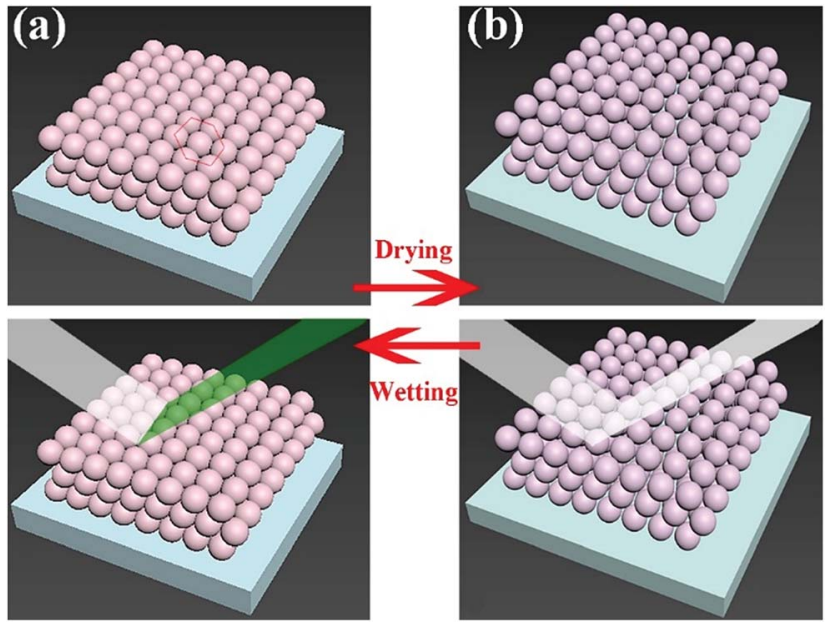

Fig. 1 A schematic diagram of the material changing its structural colour by swelling or shrinking of microspheres. nanoscale PSF microspheres were self-assembled to form blocks with a micro-nano-structure. After that, the powders were modified with triethoxy- $1 H, 1 H, 2 H, 2 H$-tridecafluoro- $n$ octylsilane (TTO), and superhydrophobic PSF was synthesized successfully. After concentration in ethyl alcohol, superhydrophobic PSF solution was coated on the glass slide, acquiring a superhydrophobic PSF surface. The PSF superhydrophobic coating possesses excellent superhydrophobicity, oleophobicity for diesel and macroscopic structural colour variation when wetted. The colour is changed when the coating is wetted and it returns to its original colour after drying. In addition, the coating can maintain its superhydrophobicity after more than 130 cycles of wetting. The coating is thermally stable below $150{ }^{\circ} \mathrm{C}$, which satisfies daily needs. The PSF superhydrophobic coating can be considered as an excellent candidate for designing wetting responsive materials, and it has enormous application potential in the fields of detection, sensing, anti-counterfeiting and security. For the first time, we present a novel and low-cost strategy to fabricate materials with both superhydrophobicity and structural colour, offering significant insights into the practical application of these functional materials.

\section{Experimental}

\section{Materials}

Reagents were obtained from the following sources: sublimed sulfur and sodium hydroxide were purchased from Aladdin (Shanghai, China); F127 was purchased from RuiDaHengHui (Beijing, China); tributyl phosphate (TBP) was purchased from Aladdin (Shanghai, China); and 1,2,3-trichloropropane (TCP) and triethoxy- $1 H, 1 H, 2 H, 2 H$-tridecafluoro- $n$-octylsilane (TTO) were purchased from Innochem (Beijing, China).

\section{Synthesis of polysulfide spheres}

PSF microspheres were synthesized by a method proposed in a previous report. ${ }^{37}$ Initially, $8 \mathrm{~g}$ sublimed sulfur were added into $250 \mathrm{~mL}$ sodium hydroxide solution which contained $10 \mathrm{~g}$ $\mathrm{NaOH}$ at $140{ }^{\circ} \mathrm{C}$ to generate $\mathrm{Na}_{2} \mathrm{~S}_{2}$ precursor solutions. The mixed solution was kept stirring for $1 \mathrm{~h}$ until all the sulfur dissolved and a reddish brown $\mathrm{Na}_{2} \mathrm{~S}_{2}$ precursor solution was obtained. In this work, all the $\mathrm{Na}_{2} \mathrm{~S}_{2}$ precursor solutions were produced under the same conditions. Then, $30 \mathrm{~mL}$ of the precursor solution were mixed with $150 \mathrm{~mL}$ of the mixed solution constituting deionized water and ethanol in a three-necked flask with a condenser pipe. The mixed solution was stirred vigorously at $70{ }^{\circ} \mathrm{C}$; then, $0.3 \mathrm{~g} \mathrm{~F} 127$ was added into the solution. A mass of bubbles was generated in the flask after vigorous stirring. Hence, $1 \mathrm{~mL}$ TBP was added into the system to eliminate bubbles, and the solution became clear and limpid. In the end, $0.75 \mathrm{~g}$ TCP was quickly added into the reaction system with vigorous stirring. A turbid emulsion was obtained $30 \mathrm{~min}$ later. In addition, the system was kept stirring at $70{ }^{\circ} \mathrm{C}$ for $6 \mathrm{~h}$. After that, a PSF colloidal sphere solution was obtained. The colloidal solution was washed with water several times by centrifugation, and all the liquid was pumped out to acquire block solids on the 
filter paper which was removed after drying. The remaining solids were dried at $60{ }^{\circ} \mathrm{C}$ for $6 \mathrm{~h}$. Moreover, the solid can present special non-iridescent structural color.

\section{Fabrication of superhydrophobic polysulfide spheres}

Some PSF sphere solids (0.5 g), which had been dried completely, were ground and put into $200 \mathrm{~mL}$ ethyl alcohol with vigorous magnetic stirring. After $30 \mathrm{~min}$, the particles were dispersed in the solution. Moreover, the mixed solution also presented a dark green color, the same as the PSF sphere solids. $500 \mu \mathrm{L}$ TTO were added into the system with a microsyringe and kept stirring for $24 \mathrm{~h}$. After that, all the liquid was pumped out by suction filtration from the system. The remaining solids were washed with ethyl alcohol several times and redispersed into $20 \mathrm{~mL}$ ethyl alcohol in a small transparent bottle. The liquid also exhibited a dark green color after redispersion.

\section{Fabrication of the superhydrophobic polysulfide sphere coating}

The PSF superhydrophobic solution was stirred adequately in the bottle. After that, $2 \mathrm{~mL}$ of the as-prepared solution were dropped and spread out evenly onto cleaned glass substrates. After drying for $1 \mathrm{~h}$ at $60{ }^{\circ} \mathrm{C}$, the ethanol had evaporated completely, and a light brown coating with superhydrophobicity was formed on the substrates. The thickness was determined by the quantity of the PSF spherical particle solution coated on the substrate.

\section{Characterization}

XPS was performed on an X-ray photoelectron spectrometer (Thermo Scientific ESCALAB $250 \mathrm{Xi}$ ). Measurements were made using the $\mathrm{Al} \mathrm{K} \alpha$ line as the excitation source. Fourier transform infrared spectroscopy (FTIR) was performed using a Thermo Scientific Nicolet iS10. TG measurements were done with a NETZSCH STA $449 \mathrm{C}$ using a dynamic heating rate of $10{ }^{\circ} \mathrm{C} \mathrm{min}^{-1}$ in air. Diffuse reflectance UV/Vis spectra were obtained with a Hewlett Packard 8452A diode array spectrophotometer. Scanning electron microscopy (SEM) images were obtained using a JSM-5601LV scanning electron microscope operating at $5 \mathrm{keV}$. Samples were prepared for SEM by lightly dusting them onto adhesive carbon and affixing them to aluminum mounts. And the size distribution was measured using a Zetasizer Nano ZS. All samples were coated with 50 Å of platinum. Contact angles (CAs) were measured with a JC2000D1. Three-dimensional surface imaging of the paper was carried out by surface imaging system atomic force microscopy (AFM, CSPM 5500). Optical photographs were taken on an iPhone 7.

\section{Results and discussion}

\section{Polysulfide microspheres}

There are many studies that have reported the theory of structural colours, and they have been reviewed by our previous studies. ${ }^{\mathbf{1 5 , 1 6}}$ Light with a specific wavelength can be captured and located in the structure of the photonic band-gap in a photonic crystal material, and other wavelength lights will be reflected. Hence, the material can exhibit various structural colours such as metal colours, iridescence and other single colours. The wavelength of reflected light on the surface can be quantitatively calculated by Bragg's law and Snell's law (eqn $(1)):^{52}$

$$
m \lambda=2 d_{h k l} \sqrt{n^{2}-\sin \theta^{2}}
$$

where $\lambda$ is the position of the PBG, $n$ is the refractive index of the material, and $n_{\mathrm{PSF}}=1$ 1.65. $\theta$ is the included angle between the incident light and the surface, $m$ is the diffraction series, " $d_{h k l}$ " is the spacing of diffraction layers for the FCC packing structure shown in the material which can be calculated using eqn (2):

$$
d_{h k l}=\frac{D \sqrt{2}}{\sqrt{\left(h^{2}+k^{2}+l^{2}\right)}}
$$

where $D$ is the space between adjacent pore layers and can also be seen as the diameter of the particle and $h, k$, and $l$ are parameters which accord with the Miller indices of a given diffraction plane.

Structural color can be attributed to the interaction between light and periodically organized architectures. Colloidal microspheres distribute uniformly on the surface to generate a photonic band gap structure. When the structure is exposed to light, a specific wavelength of incident light will be located in the structure, and others will be reflected. Therefore, the surface can present special structural color. Judging from Bragg's law and Snell's laws, the color is affected by the refractive index of the material, angle of incidence and particle spacing. We can modulate these specific parameters to acquire special structural colors. Hence, it is possible to select structural colours by controlling the size of PSF spheres and the distance between each of the particles. The size of PSF spheres can be controlled by adjusting the ratio of ethyl alcohol and deionized water. ${ }^{37}$ And the distance between each of the particles can also be controlled by liquid absorption or dehydration of the spheres. As shown in Fig. 1(a) and (b), the distance between each of the particles will diminish by inflation when the spheres absorb liquid. Due to the infiltration of liquid, each microsphere is full of ethanol. At this time, the reflectance of this material is changed and can be calculated using eqn (1). And the surface can exhibit a single structural colour. But as the liquid is drying, the spheres will shrink and the distance between each of the spheres will increase. Hence, the surface cannot exhibit structural colour. Hence, PSF sphere surfaces, which can present a single structural colour, were fabricated in this work (Table 1). The asprepared emulsion was filtered by vacuum filtration, all liquid

Table 1 Different ratios of deionized water and ethyl alcohol to fabricate PSF microspheres

\begin{tabular}{llllll}
\hline No. & $\begin{array}{l}\text { The } \mathrm{Na}_{2} \mathrm{~S}_{2} \text { precursor } \\
\text { solution }(\mathrm{mL})\end{array}$ & $\begin{array}{l}\text { Deionized } \\
\text { water }(\mathrm{mL})\end{array}$ & $\begin{array}{l}\text { Ethyl alcohol } \\
(\mathrm{mL})\end{array}$ & $\begin{array}{l}\mathrm{F}-127 \\
(\mathrm{~g})\end{array}$ & $\begin{array}{l}\text { TCP } \\
(\mathrm{g})\end{array}$ \\
\hline I & 30 & 150 & 30 & 0.3 & 0.75 \\
II & 30 & 108 & 42 & 0.3 & 0.75 \\
III & 30 & 96 & 54 & 0.3 & 0.75
\end{tabular}


was removed from the system, and PSF spheres remained and distributed on the filter paper uniformly, as shown in Fig. 2(a) and (c). The single structural colour exhibited on the filter papers is also present on the bottom of the centrifuge tube after centrifugation (Fig. 2(b)). The filter papers present green (I), red (II) and violet (III) colors when the three kinds of PSF spheres with different sizes are arranged on the surface, respectively. Sizes of PSF spheres were measured with a Zetasizer Nano ZS (Fig. 2(d)), and with increasing PSF sphere size, the structural colour was transformed from green to red to violet.

In addition, the microspheres of $180 \mathrm{~nm}$ (green), $240 \mathrm{~nm}$ (red) and $310 \mathrm{~nm}$ (violet) diameters mainly distribute on the filter paper, which almost accord with Bragg's law and Snell's laws. Judging from the SEM images of the filter paper (Fig. 2(e)), countless PSF spheres were distributed on the filter paper, almost covering the paper structure completely. Spheres were uniformly distributed on the paper surface with a hexagonal closest packed structure. On the other hand, some defects were created during the packing process, but the structure retained periodic permutations which is the required condition for the generation of structural colour. Superhydrophobic polysulfide powders with non-iridescent colour were prepared.

Based on the classical model of superhydrophobicity, surface roughness and chemical composition are essential factors which together determine the wettability properties. ${ }^{9-11}$ Hence, it is necessary to increase surface roughness and decrease surface energy for the surface wettability transformation of hydrophilic to superhydrophobic. Considering that structural colours require an orderly and regular distribution of particles, surface roughness requires an appropriate increase to guarantee that the surface presents not only super- hydrophobicity but also structural colours. An infinitesimally small amount of triethoxy- $1 H, 1 H, 2 H, 2 H$-tridecafluoro- $n$-octylsilane (TTO) was used to modify PSF powders. As shown in Fig. 3(a), PSF powders exhibited a single structural colour and the colour was disparate due to the difference in PSF microsphere sizes. The powders were added into $0.25 \%$ TTO solution and kept stirring for $24 \mathrm{~h}$. The solutions after the reaction and concentration were stored in three small transparent bottles, respectively, as shown in Fig. 3(b). And each bottle also exhibits a specific single structural

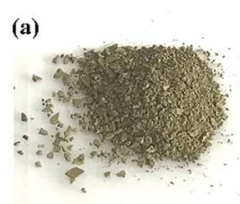

Green
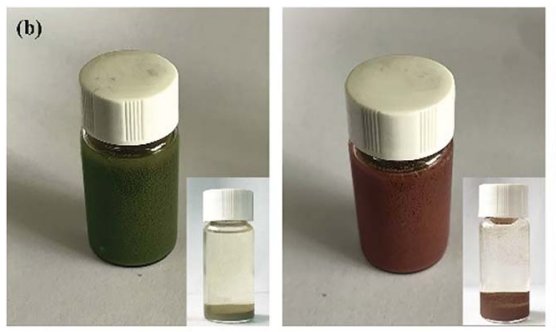

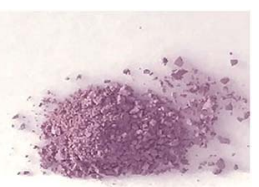

Violet
Fig. 3 (a) The images of I green, II red and III violet PSF particles. (b) The solution of the modified particles. The solution also exhibits the same structural colour as that of the particles after stirring. And the bottom right corner of each of the images shows the status of precipitation of the particles after being left to stand for a while. (a)

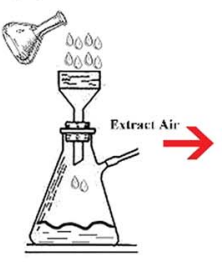

(e)

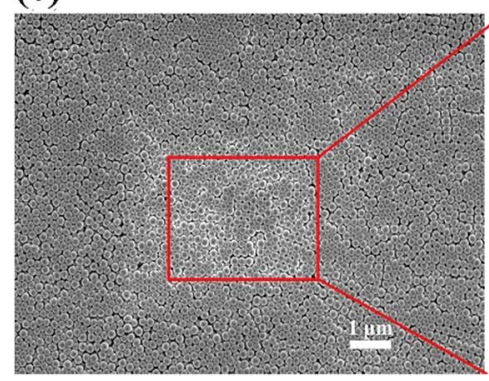

(b) After Centrifuge

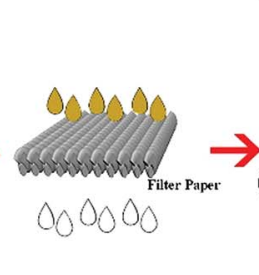

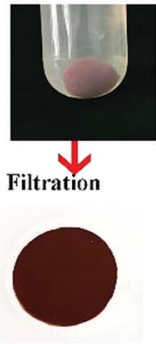

II Red

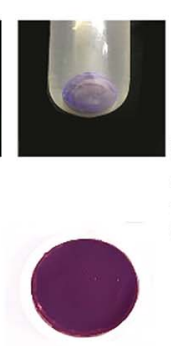

III Violet (d)

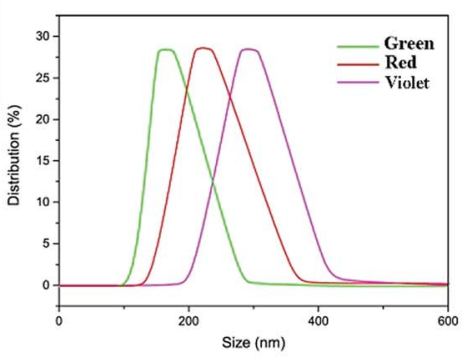

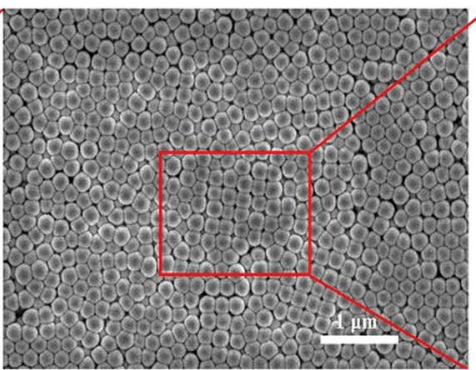

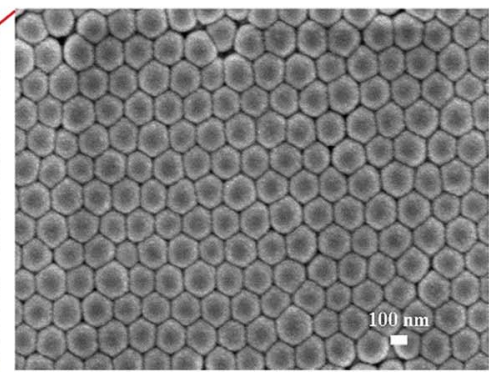

Fig. 2 (a) A schematic diagram of the preparation process to fabricate the PSF coating on filter paper. (b) The sediment of PSF microspheres on the bottom of centrifuge tubes. And I, II and III present different structural colours (I green, II red and III violet). (c) Different size PSF microspheres deposited on filter paper (I green, II red and III violet). (d) Size distribution of I, II and III PSF microspheres. (e) SEM images of II red PSF microspheres deposited on filter paper. 
colour which is similar to the PSF powders' colour before the reaction. That can be attributed to the low dispersibility of the modified particles. Hence, the particles can retain their aggregation structure in ethyl alcohol without any destruction of color. Micro-sized aggregates usually precipitate; after a while, the particles will precipitate at the bottom of the bottle, as shown in the bottom right corner of each of the figures in Fig. 3(b). Hence, when using the solution, it needs to be stirred.

XPS spectra (Fig. 4(a)) of the particles demonstrated that many elements were introduced into the particles after modification with TTO. Intense peaks of F, C, $\mathrm{Cl}$ and Si elements were observed for the modified particles compared with the original ones. Furthermore, the modified particles generate a more complicated curve in the FT-IR spectra of PSF particles (Fig. 4(b)). The spectra of both modified and unmodified particles present an intense absorption peak of $\mathrm{C}-\mathrm{H}$ at $2800 \mathrm{~cm}^{-1}$. Especially, there is a weak absorption peak of S-H stretching at $2600 \mathrm{~cm}^{-1}$. The $\mathrm{C}-\mathrm{S}$ bond generated an absorption peak at $1200 \mathrm{~cm}^{-1}$. Moreover, the $\mathrm{C}-\mathrm{F}$ bond produced an absorption peak at $1050 \mathrm{~cm}^{-1}$, and the $\mathrm{C}-\mathrm{F}$ bond in the modified particles generated a stronger absorption peak than that in the original particles. After modification, there was an absorption peak at $750 \mathrm{~cm}^{-1}$ due to $\mathrm{C}-\mathrm{Cl}$. And the absorption peak at $1050 \mathrm{~cm}^{-1}$ was generated by C-F. C-Si also generated an absorption peak at $1642 \mathrm{~cm}^{-1}$. And the difference in intensity of the $-\mathrm{OH}$ absorption peak at $3400 \mathrm{~cm}^{-1}$ also demonstrated that TTO modified the PSF powders by reaction with $-\mathrm{OH}$. In conclusion, the FT-IR and XPS spectra demonstrated that TTO modified the PSF powders successfully in the reaction process. And the modified particles possess a massive amount of $-\mathrm{Si}\left(\mathrm{CF}_{3}\right)_{3}$ which endows the surface with superhydrophobicity and superoleophobicity of diesel compared to the unmodified particles.

The TG curves (Fig. 4(c)) demonstrated that the modification with TTO did not have any influence on the thermodynamic properties of the PSF powders. An infinitesimally small amount of TTO was used during the reaction. Therefore, the thermodynamic properties were not affected by modification. And the XRD patterns (Fig. 4(d)) also confirmed that the crystal form did not transfer after modification. Both original particles and modified particles were amorphous and exhibited a smaller percentage of microsphere contraction and little warping on the substrate. However, the smaller percentage of contraction can suitably transform the structural colour of the surface when drying or wetting. Therefore, it is an excellent candidate for designing a responsive surface. For standardization of the structural colour expression, the reflection spectra of the modified PSF particles when generating structural color (Fig. 4(e)) were measured. And data of the modified coating were converted into Commission Internationale deL'Eclairage (CIE) chromaticity, which is shown in Fig. 4(f). The CIE chromaticity diagram was used to visualize the variation of structural colour, and it is important for the study of the structural discolouration of surfaces.

Particles with a single structural colour possess broad prospects in painting and decoration. And superhydrophobicity also extends the application fields of particles and endows the coating with responsiveness for the liquid.

\section{Superhydrophobic surface with non-iridescent colour}

The superhydrophobic PSF coating solution was prepared and stored in a glass bottle (Fig. 3(b)). The solution also exhibited a single structural colour which is same as the colour that the PSF powders presented. $5 \mathrm{~mL}$ of the solution was dropped and spread out evenly on cleaned glass substrates. After drying for $6 \mathrm{~h}$ at $60{ }^{\circ} \mathrm{C}$, the ethanol evaporated completely and the superhydrophobic coating was formed on the substrates, which are displayed in Fig. 5(a). The coating did not exhibit the same single structural colour as that of the particles (Fig. 3(a)). (a)

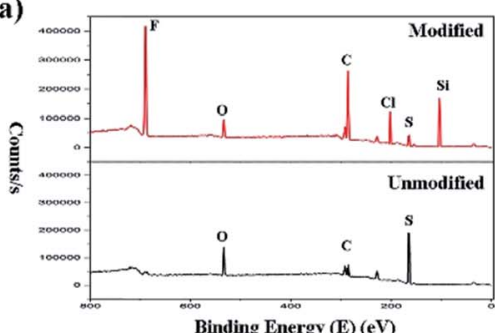

(d)

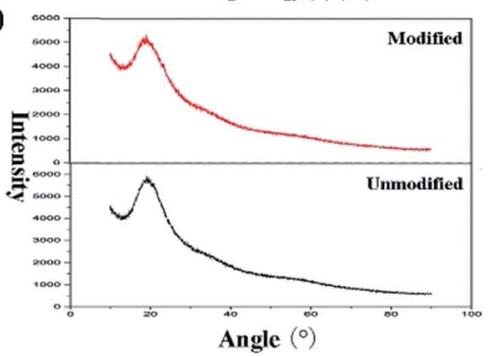

(b)

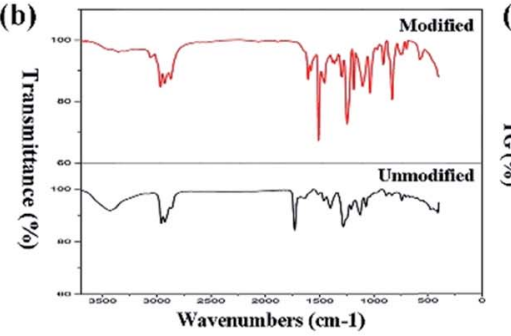

(e)

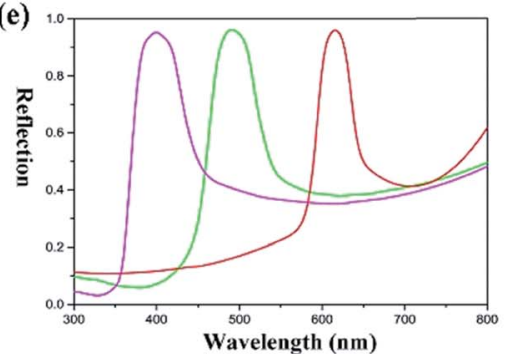

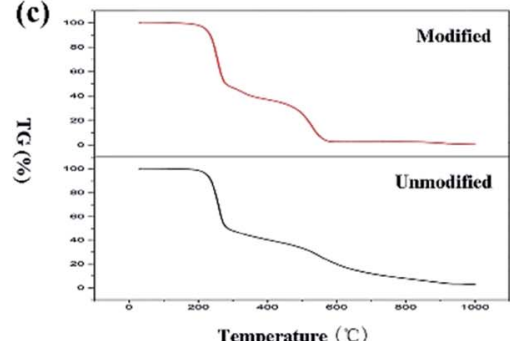

(f)

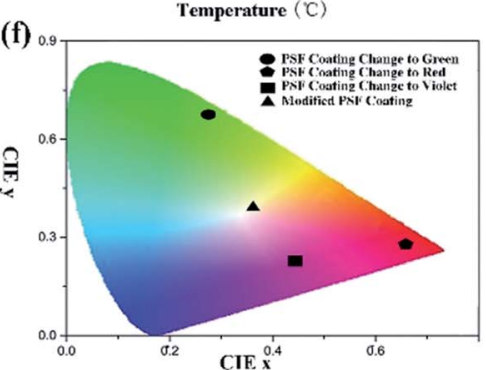

Fig. 4 (a) XPS spectra, (b) FT-IR spectra, (c) TG curves and (d) XRD patterns of the original PSF particles and modified particles. (e) The reflection spectra of the modified PSF particles when generating structural color. (f) CIE chromaticity diagram for the green, red, and violet color changes of the modified PSF coatings. 

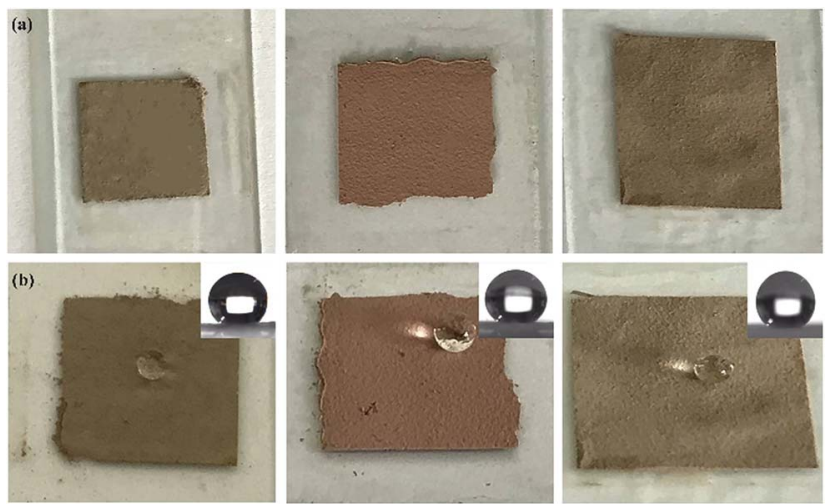

(c)
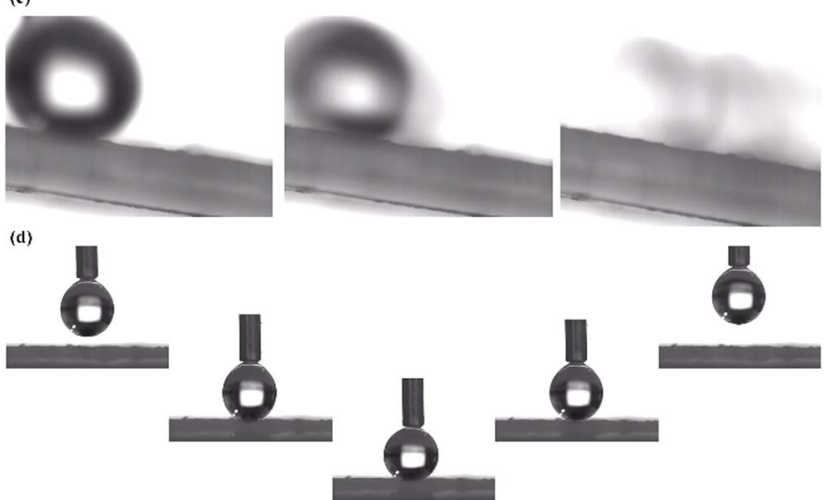

Fig. 5 (a) Optical photographs of no. I, II and III solutions coated on a glass slide. (b) Optical photographs of a liquid droplet distributed on the coating and CA images of I, II and III coatings (in the top right corner). (c) Images of a liquid droplet rolling on the surface (sliding angle $\leq 5^{\circ}$ ). (d) Images of the surface with low adhesion for the droplet.

However, the coating exhibited excellent super-hydrophobicity $\left(\mathrm{CA} \geq 150^{\circ}\right.$ and sliding angle $\leq 5^{\circ}$ ), as shown in Fig. $5(\mathrm{~b})$ and (c). The surface presented strong repellence against water. The droplet can easily be removed and rolled without any resistance, which is demonstrated in Fig. 5(d), where a droplet was lowered down and brought into contact with the surface. But the droplet still maintained its globular shape throughout the process. When the syringe with the droplet at the tip was lift up, the droplet remained at the tip and left the surface in a whole without any shape change.

As shown in the optical photographs and SEM images of the coating prepared from the original green particles and modified particles (Fig. 6(a)), the superhydrophobic surface did not exhibit the green structural colour that was presented by the original particles. The SEM images of the original coating (Fig. 6(b)) revealed that the original PSF spheres were distributed uniformly and were well-structured due to the capillary force of liquid evaporation. Countless homogeneous PSF spheres were arranged on the slide substrate uniformly and distributed on the substrate by hexagonal closest packing which led to a PBG structure. Because of this tight arrangement, the surface exhibited a single macroscopic green structural colour. Moreover, it was hydrophilic, and could be wetted by a liquid (Fig. 6(a)). The coating composed of modified PSF particles presented excellent superhydrophobicity, which is revealed by Fig. 5(b) and 6(c). The SEM images (Fig. 6(d)) were different from those in Fig. 6(b). This can be attributed to particle formation. Countless microspheres aggregated and generated particles with a micro-nano-structure before being modified by TTO.

Therefore, the particles can maintain their structural colour in solution, as is displayed in Fig. 3(b); nonetheless, they cannot generate enough roughness, as shown in Fig. 6(e). Fig. 6(d) clearly demonstrates that the coating is composed of polymeric microspheres and generates micro-nano-roughness on the substrate. Comparing the AFM images of the original and modified surfaces (Fig. 6(e)), the modified surface is found to be rougher than the original one. And the value of roughness of the modified surface is also higher. Considering that the Marmur state combines the Wenzel state and Cassie-Baxter state, ${ }^{16}$ some air can be trapped in grooves of the rough surface when a droplet comes into contact with the surface, as simulated in Fig. 6(f). These grooves can also be discovered in the AFM images (Fig. 6(e)). Also, in the surface composed of the original PSF powders, these structures can be obtained. However, the surface presented hydrophilia. That is because it exhibits nanoscale roughness and there are no hydrophobic groups in the molecule. Due to the particle aggregation and modification by reaction with TTO, the surface composed of modified PSF powders generates greater roughness, and numerous hydrophobic groups were introduced into the molecular chain. Hence, the surface can obtain excellent superhydrophobicity.

As one of the applications of a superhydrophobic surface, selfcleaning is necessary for the coating to survive in natural environments. Dust is one of the major contaminants that can pollute and damage the functional surface. And self-cleaning is important for the surface to protect its functionality. Therefore, dust was used in this study to act as a contaminant. The whole process is presented in Fig. 6(g). A certain amount of dust was placed on the surface. After that, a water column was injected onto the surface. When a droplet made contact with the dust, the dust adhered to the droplet surface and rolled down with the droplet. Then the surface cleanness was restored without any dust on it. And in the whole process, the surface was neither wetted nor cracked by the injection of the water column, which demonstrates that the coating possesses a certain degree of mechanical strength.

In addition, the thickness of the coating (II red) was measured, and the cross-sectional SEM images of the coating on the glass substrate are shown in Fig. 6(h)-(j). The thickness can be modulated by adjusting the amount of solution. The coated area was limited to $2 \mathrm{~cm} \times 2 \mathrm{~cm}$. And 1.5, 2 and $2.5 \mathrm{~mL}$ of the asprepared solution were dropped and spread out evenly onto cleaned glass substrates, respectively. And the thickness of the as-prepared coating can be clearly measured from the SEM images. The coating thicknesses when using $1.5,2$ and $2.5 \mathrm{~mL}$ solution are $8.875 \mu \mathrm{m}, 10.066 \mu \mathrm{m}$, and $15.657 \mu \mathrm{m}$, respectively. With increasing solution volume, the thickness of the coating increases. As shown in the top right corner of Fig. 6(h)-(j), the CA is almost $150^{\circ}$, and it cannot be affected by the thickness. The reflection spectra are shown in Fig. 6(k). They demonstrate that the structural color cannot be affected by the thickness either. 

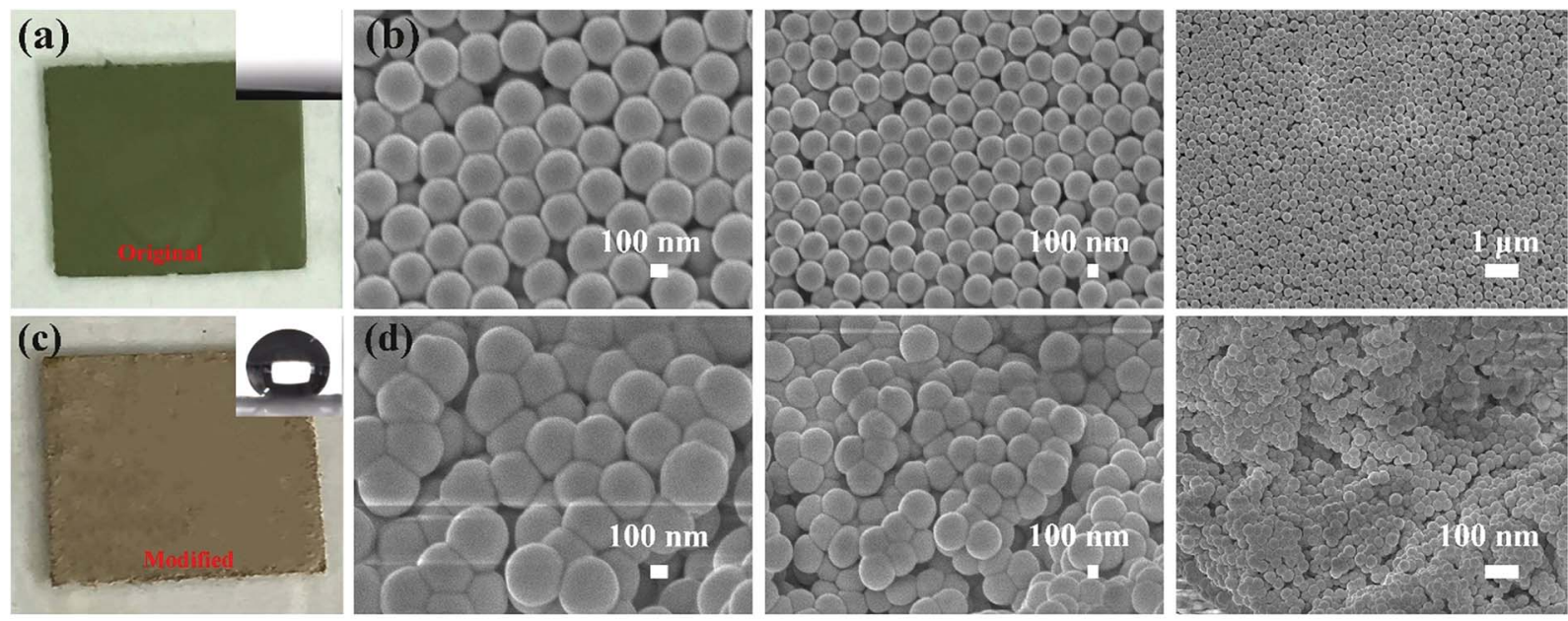

(e)

(f)

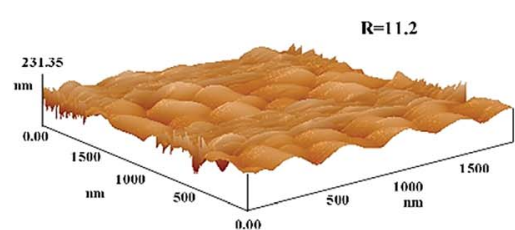

Original
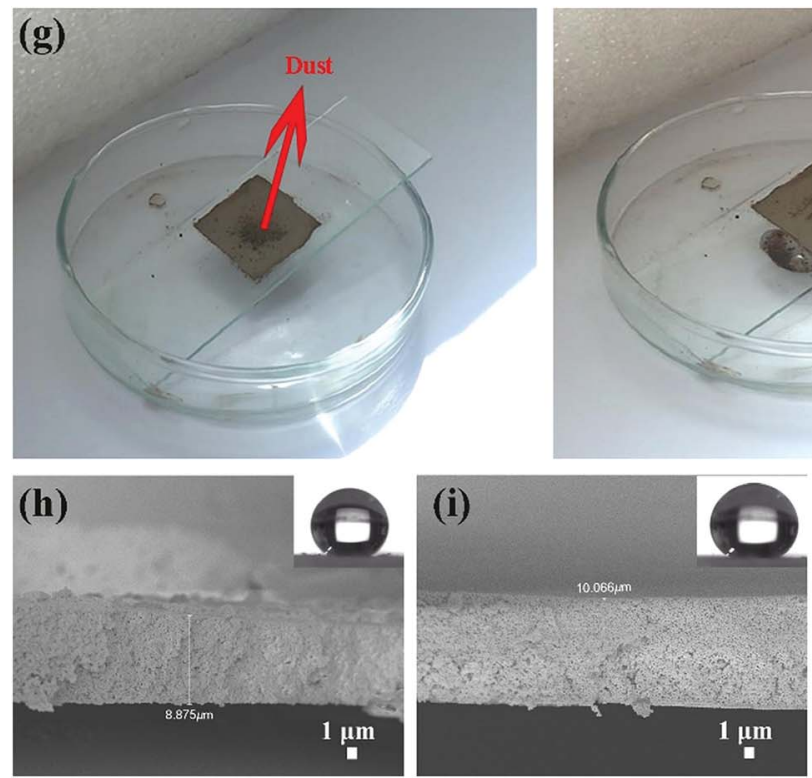

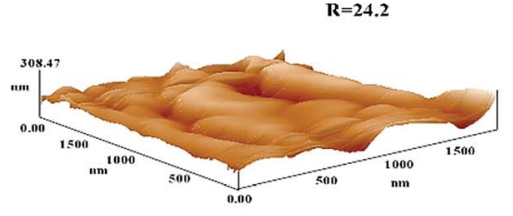

Modified
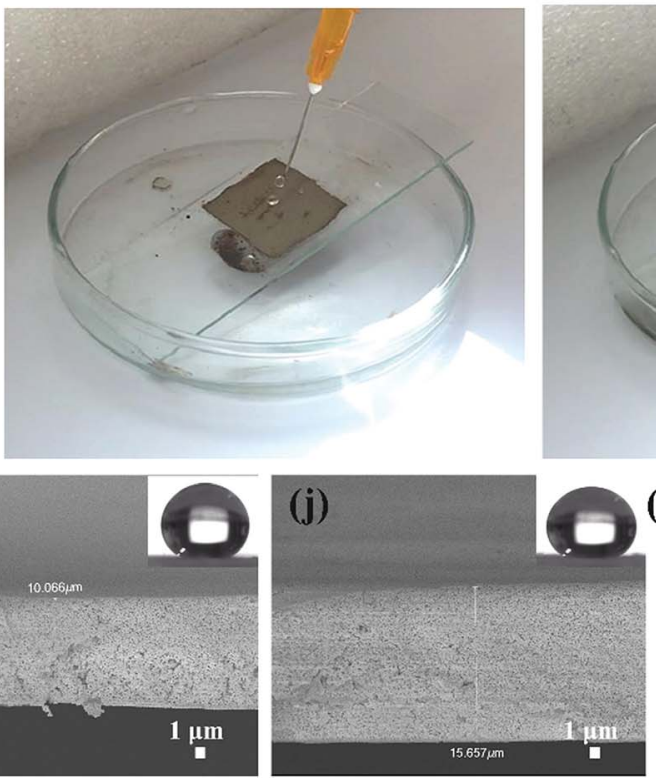

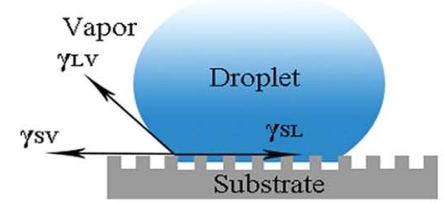

Marmur State
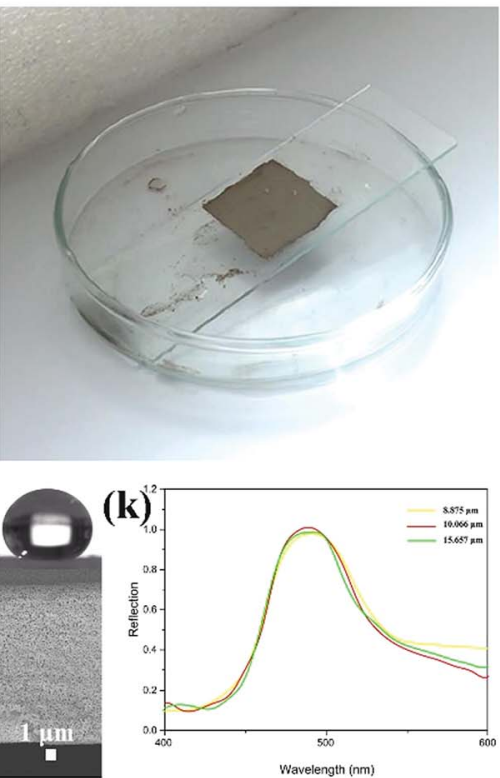

Fig. 6 (a) The optical photograph and CA image of the coating composed of the original PSF particles. (b) SEM images of the coating composed of the original PSF particles. (c) The optical photograph and CA image of the coating (I green) composed of the modified PSF particles. (d) SEM images of the coating composed of the modified PSF particles. (e) AFM images of the coatings composed of the original PSF particles and modified PSF particles. (f) Schematic diagram of the Marmur state. (g) Self-cleaning experiment of the coating composed of the modified PSF particles. ( $h$-j) Cross section of the coating (II red) with different thicknesses: (h) $8.875 \mu \mathrm{m}$, (i) $10.066 \mu \mathrm{m}$, and (j) $15.657 \mu \mathrm{m}$. The top right corner presents the CA image of the coating. (k) The reflection spectra of the II red sample with different thicknesses: (h) $8.875 \mu \mathrm{m}$, (i) $10.066 \mu \mathrm{m}$, and (j) $15.657 \mu \mathrm{m}$.

\section{Responsive and repeatable structural colour conversion by liquids}

The as-prepared coating possesses a macroscopic colour response for liquids which can wet the surface. That is attributed to the swelling or shrinkage of microspheres which has been mentioned in the previous section (Fig. 1). And the coating can repeatedly respond to the stimulation of liquids, even different kinds of liquids. The response process is presented in Fig. 7(a). A droplet wetted the surface and generated a localized wet spot on the surface. The area exhibited a special single structural colour under visible light. (In Fig. 7(a), green colour was chosen as an illustration.) After the evaporation of the 


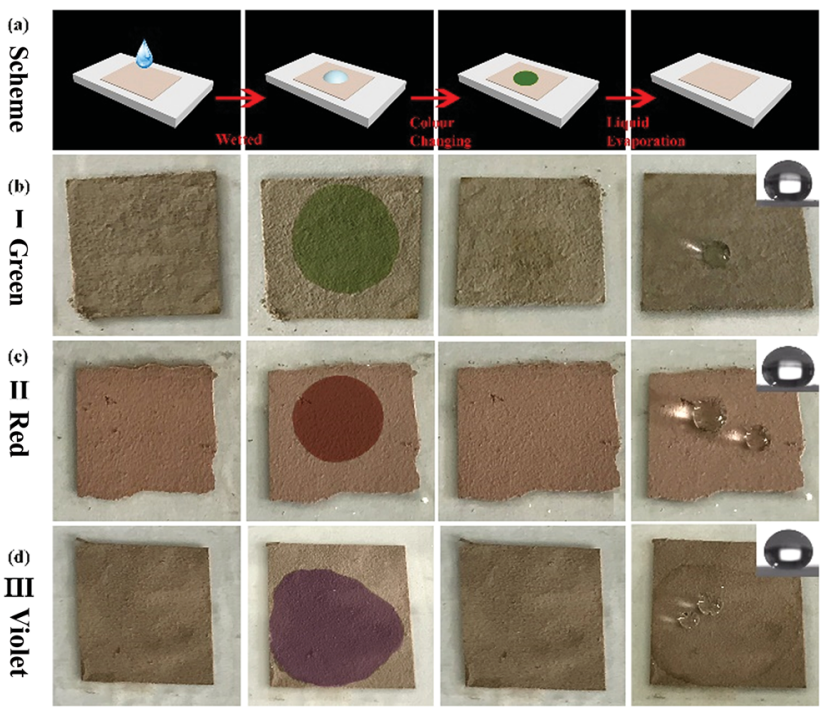

Fig. 7 (a) Schematic diagram of the colour variation of the coating wetted by a droplet. $(b-d)$ The optical photographs of colour variation after wetting of no. I, II and III superhydrophobic PSF coatings. From left to right, the images present the original coating, the coating wetted by ethyl alcohol, and the coating after drying. The rightmost images are the CA images and optical photographs of the droplets distributed on the surface.

liquid, the colour faded away, and the coating returned to its original colour. As shown in Fig. 7(b)-(d), all the coating samples presented a macroscopic colour response for ethyl alcohol. When a drop of ethyl alcohol was dropped on the surface, the coating generated high-contrast colour variation and exhibited structural colours which were similar to the colours of the original particles (Fig. 3). After the evaporation of the liquid, the coatings returned to their original colour and also possessed excellent superhydrophobicity and water repellency $\left(\mathrm{CA} \geq 150^{\circ}\right)$, as shown in the top right corner of Fig. 7(b)(d). Moreover, the reflectance at this time can also be calculated. Judging from eqn (1) and the parameters of Fig. 2(d) and 4(e), the reflectance at this time can be calculated, and the valve is 1.513.

Considering the degree of colour variation, the no. I sample was chosen to perform various performance tests because of the bright colour variation. Firstly, the thermostability of the coating was tested. The no. I sample was put into a muffle furnace and heated for $2 \mathrm{~h}$ at $20,30,40, \ldots, 150{ }^{\circ} \mathrm{C}$, respectively. After cooling, its superhydrophobicity and colour variation were tested. And the whole test process is demonstrated in Fig. 8(b). The thermostability of the coating is shown in Fig. 8(a). Judging from the TG curves of the particles (Fig. 4(c)), the test temperature range was maintained between $20^{\circ} \mathrm{C}$ and $150^{\circ} \mathrm{C}$. Fig. 8(a) demonstrates that the coating possessed a certain degree of thermostability, and can also retain its superhydrophobicity and responsive structural colour below $150{ }^{\circ} \mathrm{C}$. However, persulfide is unstable at high temperature and can easily be oxidized to sulfur dioxide. However, it can still retain its functionality below $150{ }^{\circ} \mathrm{C}$, which means that the coating is suitable for use in daily life. In addition, the responsive performance can aid the detection of the liquid by the naked eye. Moreover, we tested the responses of the coating for several other liquids. To our astonishment, all three coatings (I, II and III) presented not only excellent superhydrophobicity, but also a certain degree of oleophobicity. As shown in Fig. 8(c) and (d), five kinds of liquid were chosen to test the performance. And only diesel and water could maintain a globular shape on the surface and presented a large CA, almost $\sim 150^{\circ}$. Other liquids such as ethyl alcohol, (a)

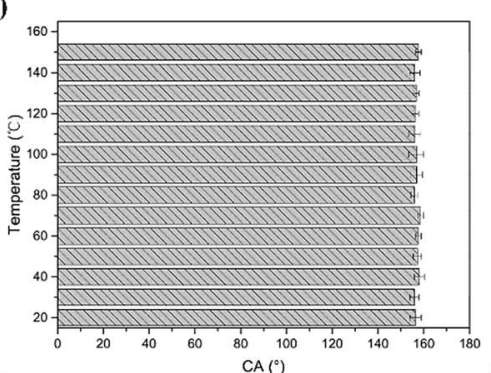

(b)

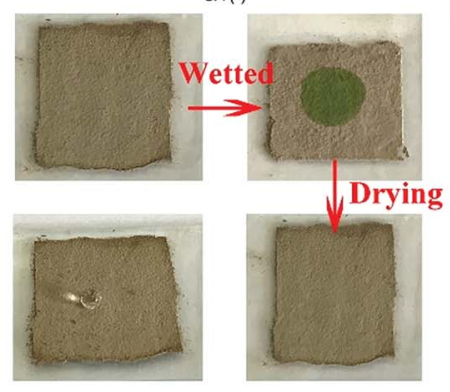

(c)

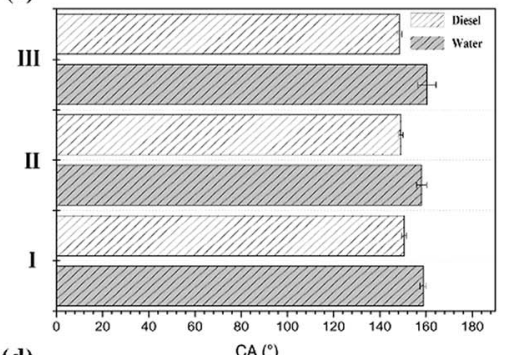

(d)

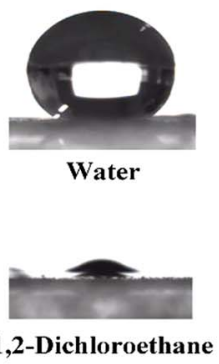

(e)

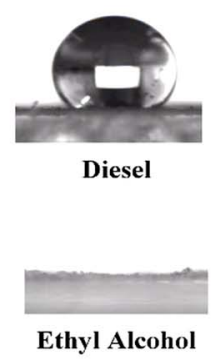

(f)
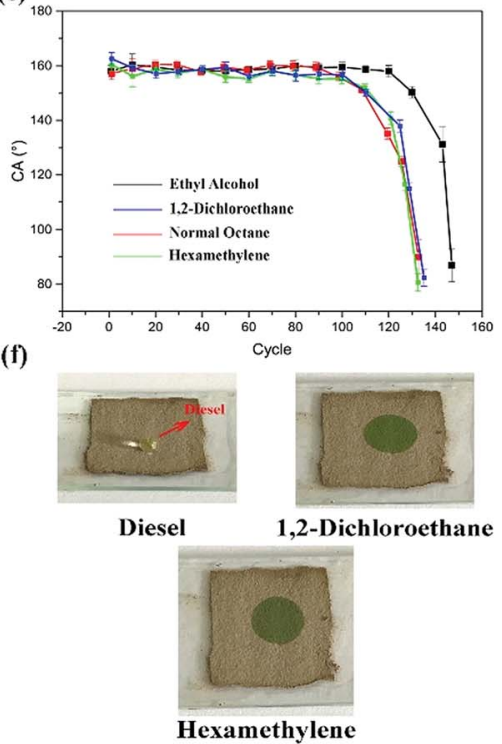

Fig. 8 (a) The thermostability of the no. I superhydrophobic coating. (b) The images of colour variation by ethyl alcohol wetting and optical photograph of a droplet distributed on the superhydrophobic surface. (c) The statistics of the water and diesel CA on the no. I, II and III superhydrophobic surfaces. (d) CA images of water, diesel, 1,2-dichloroethane and ethyl alcohol on the no. I surface. (e) The statistical data of cycle testing of the no. I coating wetted by different liquids. (f) The optical photographs of the no. I coating wetted by different liquids. 
1,2-dichloroethane, normal octane and hexamethylene wetted and spread on the surface, which means that the coating can detect liquids whether they are pure water or diesel. And when other liquids made contact with the surface, the coating exhibited a specific single structural colour (Fig. 8(f)). Cycling durability was also tested for this paper. As shown in Fig. 8(b), one cycle included three steps. First of all, the coating was wetted by a liquid and then it exhibited a specific structural colour. After that, the coating was dried at $60{ }^{\circ} \mathrm{C}$. After drying completely, the superhydrophobicity of the coating was tested. Next, these steps were repeated several times until the coating began to lose its functions. Four liquids were chosen to test the cycling durability of the coating. The results are presented in Fig. 8(e). The coating can maintain its functions for 148 cycles when wetted by ethyl alcohol. With increasing number of cycles, the surface began to be damaged and lost both its superhydrophobicity and structural colour. This can be attributed to the weak dissolution of PSF particles in ethyl alcohol. And in other oil liquids, the coating could withstand approximately 130 cycles. In this test, the coating presented better cycling durability and could respond to various liquids.

The coating is adequate for use in daily life and its macroscopic colour response behavior gives it enormous application potential in military, medicine, mechanical and other fields.

\section{Conclusions}

For the first time, a polysulfide coating with superhydrophobicity and structural colour was successfully prepared by a facile process. The PSF superhydrophobic coating possesses excellent superhydrophobicity, oleophobicity for diesel and macroscopic structural colour variation when wetted. The colour is changed when the coating is wetted and it returns to its original colour after drying. In addition, the surface presents better reusability and thermostability which satisfies various daily needs, exhibits better survivability under rainy, windy and hot natural environments. Hence, it has enormous application potential in the fields of detection, sensing, anticounterfeiting and security, and the PSF superhydrophobic coating can be considered as an excellent candidate for designing liquid responsive materials. For the first time, we present a novel and low-cost strategy to fabricate a PSF surface with both superhydrophobicity and structural colour, offering significant insights into the practical application of this functional material.

\section{Conflicts of interest}

There are no conflicts to declare.

\section{Acknowledgements}

This work is supported by the National Nature Science Foundation of China (No. 51675513, 51522510 and 51735013).

\section{Notes and references}

1 W. Cao, Y. Liu, M. Ma and J. Zhu, Colloids Surf., A, 2017, 529, 18.

2 Z. Geng, X. Yang, C. Boo, S. Zhu, Y. Lu, W. Fan, M. Huo, M. Elimelech and X. Yang, J. Membr. Sci., 2017, 529, 110.

3 Q. Shang and Y. Zhou, Ceram. Int., 2016, 42, 8706.

4 D. Nanda, P. Varshney, M. Satapathy, S. Mohapatra, B. Bhushan and A. Kumar, J. Colloid Interface Sci., 2017, 507, 397.

5 T. Chen, W. Yan, L. Hongtao, W. Zhu, K. Guo and J. Li, J. Mater. Sci., 2017, 52, 4675.

6 Z. Zuo, R. Liao, X. Zhao, X. Song, Z. Qiao, C. Guo, A. Zhuang and Y. Yuan, Appl. Therm. Eng., 2017, 110, 39.

7 W. Zhang, Z. Yu, Z. Chen and M. Li, Mater. Lett., 2012, 67, 327.

8 Y. Wang, W. Wang, L. Zhong, J. Wang, Q. Jiang and X. Guo, Appl. Surf. Sci., 2010, 256, 3837.

9 R. Wenzel, Ind. Eng. Chem., Anal. Ed., 1936, 28, 988.

10 A. Cassie and S. Baxter, Trans. Faraday Soc., 1944, 40, 546.

11 A. Marmur, Langmuir, 2003, 19, 8343.

12 J. Li, L. Shi, Y. Chen, Y. Zhang, Z. Guo, B. Su and W. Liu, J. Mater. Chem., 2012, 22, 9774.

13 Y. Si, Z. Guo and W. Liu, ACS Appl. Mater. Interfaces, 2016, 8, 16511.

14 Y. Si, H. Zhu, L. Chen, T. Jiang and Z. Guo, Chem. Commun., 2015, 51, 16794.

15 H. Fudouzi, Colloidal Photonic Crystal Films: Fabrication and Tunable Structural Color and Applications, Springer, NY, USA, 2015.

16 Z. Wang and Z. Guo, Chem. Commun., 2017, 53, 12990.

17 Z. Wang and Z. Guo, Journal of Bionic Engineering, 2018, 15.1, 1.

18 Y. Zhang, X. Li, L. Gao, J. Qiu, L. Heng, B. Tang and L. Jiang, ChemPhysChem, 2014, 15, 507.

19 H. Wang, X. Gu, R. Hu, J. Lam, D. Zhang and B. Tang, Chem. Sci., 2016, 7, 5692.

20 Z. Cai and S. Asher, Chem. Sci., 2016, 7, 4557.

21 J. Li and Z. Yang, Chem. Commun., 2016, 52, 284.

22 O. Poncelet, G. Tallier, P. Simonis, A. Cornet and L. Francis, Bioinspiration Biomimetics, 2015, 10, 026004.

23 L. Zhang, Z. Xiong, L. Shan, L. Zheng, T. Wei and Q. Yan, Small, 2015, 11, 4910.

24 B. Tang, C. Wu, T. Lin and S. Zhang, Dyes Pigm., 2013, 99, 1022.

25 Z. Gu, H. Chen, S. Zhang, L. Sun, Z. Xie and Y. Ge, Colloids Surf. A, 2007, 302, 312.

26 M. Egen and R. Zentel, Chem. Phys., 2004, 205, 1479.

27 B. Tang, X. Zheng, T. Lin and S. Zhang, Dyes Pigm., 2014, 104, 146.

28 J. Goodwin, Rheology of Polymer Colloids. Science and Technology of Polymer Colloids, Vol. I, Martinus Nijhoff, Boston, MA, USA, 1983.

29 Z. Yang, S. Gao, W. Li, V. Vlasko-Vlasov, U. Welp, W. Kwok and T. Xu, ACS Appl. Mater. Interfaces, 2011, 3, 1101. 
30 S. Wang, W. Li, G. Hao, Y. Hao, Q. Sun, X. Zhang and A. Lu, J. Am. Chem. Soc., 2011, 133, 15304.

31 J. Liu, S. Qiao, H. Liu, J. Chen, A. Orpe, D. Zhao and G. Lu, Angew. Chem., Int. Ed., 2011, 50, 5947.

32 A. Van Blaaderen, J. Van Geest and A. Vrij, J. Colloid Interface Sci., 1992, 154, 481.

33 W. Wang, B. Tang, W. Ma, J. Zhang, B. Ju and S. Zhang, J. Opt. Soc. Am. A, 2015, 32, 1109.

34 D. Ge, E. Lee, L. Yang, Y. Cho, M. Li, D. Gianola and S. Yang, Adv. Mater., 2015, 27, 2489.

35 E. Trofimova, D. Kurdyukov, S. Yakovlev, D. Kirilenko, Y. Kukushkina, A. Nashchekin, A. Sitnikova, M. Yagovkinaa and V. Golubev, Nanotechnology, 2013, 24, 155601.

36 J. Kelly, A. Shukaliak, C. Cheung, K. Shopsowitz, W. Hamad and M. Mac Lachlan, Angew. Chem., Int. Ed., 2013, 52, 8912.

37 F. Li, B. Tang, S. Wu and S. Zhang, Small, 2017, 13.3, 1602565.

38 B. Tang, C. Wu, T. Lin and S. Zhang, Dyes Pigm., 2013, 99.3, 1022.

39 L. Wang, S. Zhang, J. Lutkenhaus, L. Chu, B. Tang, S. Li and W. Ma, J. Mater. Chem. C, 2017, 5.32, 8266.
40 J. Joannopoulos, S. Johnson, J. Winn and R. Meade, Photonic Crystals: Molding the Flow of Light, Princeton University Press, NJ, USA, 2011.

41 U. Jeong, J. Kim, Y. Xia and Z. Li, Nano Lett., 2005, 5, 937.

42 U. Jeong and Y. Xia, Angew. Chem., Int. Ed., 2005, 44, 3099.

43 Y. Zhang, B. Dong, A. Chen, X. Liu, L. Shi and J. Zi, Adv. Mater., 2015, 27, 4719.

44 M. Xiao, Y. Li, M. C. Allen, D. D. Deheyn, X. Yue, J. Zhao, N. C. Gianneschi, M. D. Shawkey and A. Dhinojwala, ACS Nano, 2015, 9(5), 5454.

45 M. Kohri, Y. Nannichi, T. Taniguchi and K. Kishikawa, J. Mater. Chem. C, 2015, 3, 720-724.

46 Y. Zhao, Z. Xie, H. Gu, C. Zhu and Z. Gu, Chem. Soc. Rev., 2012, 41, 3297.

47 F. Barrows and M. Bartl, Nanomater. Nanotechnol., 2014, 4, 1. 48 J. Sun, B. Bhushan and J. Tong, RSC Adv., 2013, 3, 14862.

49 A. Dumanli and T. Savin, Chem. Soc. Rev., 2016, 45, 6698.

50 F. Wang, X. Zhang, L. Zhang, M. Cao, Y. Lin and J. Zhu, Dyes Pigm., 2016, 130, 202-208.

51 Y. Meng, B. Tang, J. Cui, S. Wu, B. Ju and S. Zhang, Adv. Mater. Interfaces, 2016, 3, 1600374.

52 A. Richel, N. Johnson and D. McComb, Appl. Phys. Lett., 2000, 76, 1816-1818. 